\title{
SARS-CoV-2 seroprevalence among blood donors in Québec, and analysis of symptoms associated with seropositivity: a nested case-control study
}

\author{
Antoine Lewin ${ }^{1,2} \cdot$ Roseline Therrien ${ }^{3} \cdot$ Gaston De Serres ${ }^{4,5} \cdot$ Yves Grégoire $^{6} \cdot$ Josée Perreault $^{6} \cdot$ Mathieu Drouin $^{6}$. \\ Marie-Josée Fournier $^{6} \cdot$ Tony Tremblay $^{6} \cdot$ Julie Beaudoin $^{3} \cdot$ Guillaume Beaudoin-Bussières $^{7,8}$ • Jérémie Prévost ${ }^{7,8}$. \\ Gabrielle Gendron-Lepage $^{7}$. Andrés Finzi ${ }^{7,8} \cdot$ France Bernier $^{3} \cdot$ Renée Bazin $^{6} \cdot$ Marc Germain $^{6} \cdot$ Gilles Delage $^{1}$ (D)
}

Received: 17 February 2021 / Accepted: 15 April 2021 / Published online: 17 May 2021

(C) The Canadian Public Health Association 2021

\begin{abstract}
Objectives A substantial proportion of individuals infected with SARS-CoV-2 do not experience noticeable symptoms typical of COVID-19. Our objectives were to evaluate the impact of the first wave of the pandemic in Québec by measuring SARS-CoV-2 antibody seroprevalence in a convenience sample of healthy blood donors and to study the association between seropositivity and the occurrence of COVID-19 symptoms.

Methods The study design was a cross-sectional serological survey with a nested case-control study. Residual blood samples from donations collected between May 25 and July 9, 2020 (well before vaccination rollout) in the province of Québec were tested for anti-Spike RBD antibodies by ELISA. Seropositive donors and a control group of seronegative donors were questioned about prior COVID-19 symptoms. All qualified blood donors were eligible for participation.

Results A total of 7691 blood donors were included in the study. After adjustments, the seroprevalence rate was $2.2 \%$ (95\% CI 1.9-2.6). Seropositive donors reported one or more symptoms in a proportion of 52.2\% (95\% CI 44.2-60.1); this proportion was $19.1 \%$ (95\% CI 13.4-26.1) among seronegative donors, suggesting that approximately 50-66\% of all infections were asymptomatic. Univariate analysis of associations between symptoms and seropositivity revealed that except for rhinorrhea, all symptoms were significantly associated with seropositivity.

Conclusion Assuming that blood donors are fairly representative of the general adult population, this study shows that less than $3 \%$ of 18-69-year-olds have been infected during the first wave of the pandemic in the province of Québec. Our data also confirm that many infections escaped detection, including a substantial proportion that were asymptomatic.
\end{abstract}

\section{Résumé}

Objectifs Une proportion substantielle de personnes infectées par le SRAS-CoV-2 ne présentent pas de symptômes visibles typiques de la COVID-19. Nos objectifs étaient d'évaluer l'impact de la première vague de la pandémie au Québec en mesurant la séroprévalence des anticorps anti-SRAS-CoV-2 chez les donneurs de sang en bonne santé, et d'étudier l'association entre la séropositivité et la survenue des symptômes de la COVID-19.

Méthodes Le design de l'étude était une enquête de sérologie transversale avec une étude cas-témoins nichée dans la cohorte. Des échantillons de sang provenant de dons recueillis entre le 25 mai et le 9 juillet 2020 (bien avant le déploiement de la

Gilles Delage

Gilles.Delage@ hema-quebec.qc.ca

1 Héma-Québec, Affaires Médicales et Innovation, 4045 Blvd. de la Côte-Vertu, Saint-Laurent, Montréal, QC H4R 2W7, Canada

2 Faculté de médecine et des sciences de la santé, Université de Sherbrooke, Sherbrooke, QC J1H 5N4, Canada

3 Héma-Québec, Qualité et développement, Montréal, QC H4R 2W7, Canada
4 Institut National de Santé Publique du Québec, Québec, QC H2P 1E2, Canada

5 Centre de recherche du CHU de Québec-Université Laval, Québec, QC G1E 6W2, Canada

6 Héma-Québec, Affaires Médicales et Innovation, Québec, QC G1V 5C3, Canada

7 Centre de Recherche du CHUM, Montréal, QC H2X 0A9, Canada

8 Département de Microbiologie, Infectiologie et Immunologie, Université de Montréal, Montréal, QC H2X 0A9, Canada 
vaccination) dans la province de Québec ont été testés pour les anticorps anti-spicule RBD (Receptor Binding Domain) par ELISA. Les donneurs séropositifs et un groupe témoin de donneurs séronégatifs ont été interrogés sur les symptômes spécifiques à la COVID-19. Tous les donneurs qualifiés pour le don de sang étaient éligibles à participer à l'étude.

Résultats Au total, 7691 donneurs de sang ont été inclus dans l'étude. Après ajustements, le taux de séroprévalence était de 2,2\% (IC à 95\% 1,9-2,6). Les donneurs séropositifs ont signalé un ou plusieurs symptômes dans une proportion de 52,2 \% (IC à 95\% 44,2-60,1); cette proportion était de 19,1\% (IC à 95\% 13,4-26,1) parmi les donneurs séronégatifs, ce qui suggère qu'entre $50 \%$ et $66 \%$ de toutes les infections étaient asymptomatiques. Une analyse univariée des associations entre les symptômes et la séropositivité a révélé qu'à l'exception de la rhinorrhée, tous les symptômes étaient significativement associés à la séropositivité. Conclusion En supposant que les donneurs de sang sont assez représentatifs de la population adulte générale, cette étude montre que moins de $3 \%$ des 18-69 ans ont été infectés lors de la première vague de la pandémie dans la province du Québec. Nos données confirment également que de nombreuses infections n'ont pas fait l'objet d'un test moléculaire de dépistage, y compris une proportion importante qui était asymptomatique.

Keywords SARS-CoV-2 $\cdot$ Seroprevalence $\cdot$ COVID-19 $\cdot$ Asymptomatic $\cdot$ Signs and symptoms $\cdot$ ELISA RBD

Mots-clés SRAS-CoV-2 $\cdot$ séroprévalence $\cdot$ COVID-19 $\cdot$ asymptomatique $\cdot$ signes et symptômes $\cdot$ ELISA RBD

\section{Introduction}

The COVID-19 pandemic caused by SARS-CoV-2 is a public health crisis of global proportions (Worldometer, 2020; Center for Systems Science and Engineering (CSSE), 2020; European Centre for Disease Prevention and Control (ECDC), 2020). In most cases, the infection causes mild to moderate symptoms but can result in significant morbidity and mortality in those with pre-existing conditions and in the elderly; others may become infected and have no symptoms (Mizumoto et al., 2020; Wu \& McGoogan, 2020). The number of confirmed cases identified by health care systems is one indicator of the progression of the pandemic. However, the true burden of infection can be more precisely estimated by SARS-CoV-2 antibody seroprevalence in the general population. Seroprevalence studies have the potential of evaluating the proportion of infections that are missed by the health care system, including those that are asymptomatic. Several studies have been published, with varying but often low seroprevalence rates, usually below ten percent (Chen et al., 2020; Lai et al., 2020). In addition, these studies often show rates of infection that are higher than those diagnosed by PCR, confirming that a substantial proportion of infected individuals remain undiagnosed. Few studies in a general population setting have correlated the presence or absence of anti-SARSCoV-2 antibodies with the occurrence of COVID-19 symptoms (Qu et al., 2020; Zhao et al., 2020).

Québec was the province most severely hit by COVID-19 during the first wave of the pandemic, with more than $50 \%$ of all cases in the country, while representing only $23 \%$ of its population (i.e., 57,007 cases in the province of Québec, and a total of 108,829 cases in Canada as of July 15) (Institut national de santé publique du Québec, n.d.-a). The greater Montréal area, where more than half of the population of the province lives, was especially affected with a reported cumulative incidence of confirmed COVID-19 cases of 1350 per 100,000 between March and mid-July 2020.

The main goal of this study was to determine the seroprevalence of anti-SARS-CoV-2 antibodies in the Québec general population after the first wave of the pandemic, in blood samples from donors collected by Héma-Québec, the provincial blood collection agency. The dynamics of pre- and asymptomatic infections are still under scrutiny (Nogrady, 2020). Therefore, a second goal was to estimate the frequency of asymptomatic SARS-CoV-2 infections in this population.

\section{Methods}

\section{Study participants for seroprevalence study}

Residual blood samples were obtained from 7691 consenting blood donors who gave a regular blood donation at one of Héma-Québec's permanent donation centres or mobile blood drives. Donors are residents of the province of Québec, where Héma-Québec is the sole blood component supplier for the province. Ninety-five percent of donations made at selected clinics were tested and included in the study. Sample size was stratified by region, and based on an expected regional prevalence of 3\%, targeting a $95 \%$ accuracy of about $\pm 2 \%$. Prospective donors included in the study had to be free of COVID-19 symptoms in the preceding 14 days and otherwise eligible to donate according to standard criteria. Samples were collected between May 25 and July 9, 2020 from 12 of the 18 health regions of the province of Québec. A higher sampling rate was applied in regions where COVID-19 was more prevalent. Donors first learned about the study when they presented to donate.

At the time of this study, guidelines from Québec public health authorities recommended COVID-19 testing for: (1) people with symptoms; (2) people who have had close 
contact with someone who has COVID-19; and (3) people who have been asked by public health authorities to get tested.

\section{Anti-SARS-CoV-2 Spike RBD ELISA}

An in-house ELISA targeting the RBD of the SARS-CoV-2 Spike protein was adapted from an assay designed to establish seropositivity of COVID-19 convalescent plasma (Perreault et al., 2020). SARS-CoV-2 Spike RBD was chosen over other SARS-CoV-2 antigens because of its low homology with common cold human coronaviruses, thus limiting cross-reactivity (Heffron et al., 2020; Prévost et al., 2020; Shrock et al., 2020). Assay design allows for the capture of the three main classes of immunoglobulins (IgG, IgA, and IgM). Plasma samples were diluted 1:100, as this dilution enabled easily distinguishing seropositive from seronegative samples with high sensitivity (98.9\%) and specificity (98.5\%) (Perreault et al., 2020). As standard procedure in a blood bank context, initially reactive samples were retested twice and were considered positive if at least two out of three assay results were above the seropositivity threshold. Positive and negative controls were included in each ELISA microtiter plate to validate assay results.

\section{Occurrence of symptoms in cases and controls}

We conducted a nested case-control study comparing all 173 antibody-positive individuals and 176 randomly selected antibody-negative control donors. Case-control study participants were notified in writing that HémaQuébec would contact them by phone in the weeks following their blood donation. The survey was non-blinded as donors and interviewers were aware of the seroprevalence results. The five interviewers involved in the study followed a standard script. Donors were queried on basic socio-demographic characteristics (sex, age, healthcare worker status, and area of residence), COVID-19-related symptoms, contacts with individuals with confirmed or suspected COVID-19, COVID-19 testing, and results (see questionnaire in Supplementary Material), for the period between March 1, 2020 and the date of donation. Mean number of days between testing and survey was 54.5 (SD 12.0) and 57.6 (SD 14.0) for cases and controls, respectively, with an overall delay of 56.1 days (SD 13.1).

\section{Statistical analyses}

Crude seroprevalence rates and their respective 95\% Clopper-Pearson confidence intervals (CI) were calculated from the proportion of study participants who had developed antibodies against SARS-CoV-2. Seroprevalence rates were then adjusted to reflect population demographics. Age and sex distribution of seropositivity within each Québec health region was determined. Population data were derived from the 2011 Québec census. The adjustment was calculated according to the following formula:

Individual adjustment factor

$$
=\text { Ppop }_{\text {region,age,sex }} / \text { Psample }_{\text {region, age,sex }}
$$

where Ppop region, age, sex and Psample region, age, sex correspond, respectively, to the proportion of the population of a given health region and the proportion of the sample for that region, stratified by age and sex. Seroprevalence results were compared with the cumulative incidence of COVID19 , as reported by Québec public health authorities as of July 20, 2020 (Institut national de santé publique du Québec, n.d.-b).

For the nested case-control study, associations between seropositivity and reported symptoms were expressed as odds ratios and their 95\% CI derived from logistic regression analyses, adjusting for age (continuous variable) and sex. We used parsimonious stepwise multivariable logistic regression to estimate the strongest association between a given combination of symptoms and seropositivity. We initially included variables that were significantly $(p \leq 0.05)$ associated with seropositivity in the univariate analysis, then each variable was iteratively excluded from the multivariable model until only those significantly associated with seropositivity remained ( $p \leq 0.05$ ), further adjusting for age and sex. The same strategy was used to estimate risk factors for SARS-CoV-2 infection.

\section{Results}

\section{Seroprevalence study}

A total of 7691 blood donors consented to participate in the study and to have a blood sample tested for the presence of anti-SARS-CoV-2 antibodies; $52.8 \%$ of these were males and $39.8 \%$ were from the Montréal-Laval area. Donors from the Montréal-Laval and Montréal surrounding regions represent $64.8 \%$ of all participants.

Of 7691 samples, 173 were found to be seropositive for antiSARS-CoV-2 Spike RBD antibodies, for a crude seroprevalence rate of $2.25 \%$ (95\% CI 1.93-2.61). After adjusting for population size, age, and sex, seroprevalence was $2.23 \%(95 \%$ CI 1.90-2.56) (Table 1). Seroprevalence rates by health region correlated well with cumulative incidence rates of confirmed COVID-19 cases, not restricted for the age range of donors, as reported by health authorities $(r=0.80 ; p=0.0017)$. The 
Table 1 Seroprevalence data (raw and adjusted for population, sex, and age) in Montréal-Laval, surrounding Montréal and Laval urban areas, and other regions

\begin{tabular}{|c|c|c|c|c|}
\hline Region & $\begin{array}{l}\text { Number of antibody-positive } \\
\text { donors/total number of tested donors }\end{array}$ & $\begin{array}{l}\text { Raw seroprevalence } \\
\%(95 \% \mathrm{CI})\end{array}$ & $\begin{array}{l}\text { Adjusted seroprevalence } \\
\%(95 \% \mathrm{CI}) \dagger\end{array}$ & $\begin{array}{l}\text { Cumulative COVID-19 } \\
\text { incidence } \\
(/ 100,000) \$\end{array}$ \\
\hline Montréal-Laval & $90 / 3061$ & $2.94(2.34-3.54)$ & $3.05(2.44-3.66)$ & 1350 \\
\hline $\begin{array}{l}\text { Surrounding Montréal-Laval } \\
\text { urban areas }\end{array}$ & $48 / 1925$ & $2.49(1.84-3.29)$ & $2.24(1.58-2.90)$ & 800 \\
\hline Other regions $\S$ & $35 / 2705$ & $1.29(0.90-1.78)$ & $1.29(0.86-1.72)$ & 220 \\
\hline Total & $173 / 7691$ & $2.25(1.93-2.61)$ & $2.23(1.90-2.56)$ & 667 \\
\hline
\end{tabular}

$†$ Seroprevalence data adjusted for population, sex, and age in each region

$\$$ As reported by Québec public health authorities as of July 20, 2020

$\S$ This includes all regions of the province of Québec except Montréal-Laval and Surrounding Montréal-Laval urban areas

cumulative incidence rate in Montréal and Laval, at 1350 cases per 100,000, was about six times higher than in health regions outside of the greater Montréal area (220 cases per 100,000). However, the adjusted seroprevalence rate in Montréal and Laval at $3.05 \%$ (95\% CI 2.44-3.66) was only about 2.5 -fold higher than in regions outside the greater Montréal area (1.29\%; 95\% CI 0.86-1.72).

Women had a higher adjusted seroprevalence rate $(2.51 \%$; 95\% CI $2.00-3.02)$ than men $(1.97 \%$; 95\% CI 1.54-2.41) but the difference was not statistically significant. The adjusted seroprevalence appeared to decrease with age, but the trend, using the Cochran-Armitage test, was non-significant (18-24 years: $2.57 \%$ (95\% CI $1.70-3.44)$; 25-39 years: $2.36 \%$ (95\% CI 1.69-3.02); 40-59 years: $2.29 \%$ (95\% CI 1.74-2.83); 60 69 years: $1.68 \%$ (95\% CI $1.03-2.33)$ ).

\section{Nested case-control study of association between symptoms and seroprevalence}

Of the 173 antibody-positive donors, 161 (93.0\%) agreed to participate in the telephone survey; for controls, 162/176 $(92.0 \%)$ answered the survey. Among antibody-positive donors, $52.2 \%$ ( $n=84 ; 95 \%$ CI 44.2-60.1) reported at least one of 11 COVID-related symptoms between March 1 and 14 days before their donation date; this proportion was $19.1 \%$ ( $n=31$; 95\% CI 13.4-26.1) among seronegative controls (Table 2). Except for rhinorrhea, all symptoms were significantly more frequent among antibody-positive donors than among controls. Symptoms most typically associated with COVID-19 (fever $\geq 38^{\circ} \mathrm{C}$ or sensation of fever/chills, generalized pain, shortness of breath/difficulty breathing, and loss or reduction in smell or taste sensation) were more strongly associated with seropositivity, and were experienced by less than $2 \%$ of antibody-negative controls. Using stepwise multivariable logistic regression, fever or sensation of fever/chills (odds ratio $10.4,95 \%$ CI 3.0-35.4) and loss or reduction in smell or taste sensation (odds ratio $14.5,95 \%$ CI 1.8-114.0) were independently associated with seropositivity. The presence of both symptoms was present in 16/84 (19\%) of symptomatic antibody-positive donors, and in none of the symptomatic antibody-negative donors. The presence of at least one of these symptoms was reported by $57 \%$ of symptomatic antibody-positive donors, but by only $13 \%$ (4/31) of symptomatic antibody-negative donors.

Table 3 presents the results of additional questions that targeted symptomatic individuals, as well as those who consulted a physician for suspected or actual COVID-19 symptoms. Among antibody-positive donors, 16 (14 symptomatic and two symptom-free) consulted a physician; 70 symptomatic donors did not consult. Only three of 31 symptomatic antibodynegative control donors consulted a physician. Furthermore, 22 donors (19 antibody-positive and 3 antibody-negative) had a COVID-19 PCR test performed; 14/19 antibody-positive donors tested positive by PCR for COVID-19, but none of the three antibody-negative donors were PCR-positive. Assuming that symptoms reported by all 84 antibody-positive donors were caused by COVID-19, we infer that $83 \%$ [(84-14)/84] were not diagnosed as such at the time of infection because, as mentioned above, they did not consult a physician. If one also considers that $19.1 \%$ of antibody-negative controls had symptoms as compared with $52.2 \%$ of antibody-positive donors, a more conservative estimate could be that about one third $(52.2 \%-19.1 \%=33.1 \%)$ of antibody-positive individuals had symptoms related to COVID-19, which means that around 53 antibody-positive donors experienced COVID-19 symptoms, including the 14 individuals who consulted a physician, and that $74 \%$ of these clinical COVID-19 cases [(53 - 14)/53] did not contact public health authorities or consult a physician for COVID-19 diagnosis.

The vast majority of antibody-positive donors were unaware of how they were infected: $78.9 \%$ did not recall a contact with a COVID-19-positive individual, and $73.9 \%$ were unaware of a contact with an individual having cough or fever (Table 4). 
Table 2 Association between symptoms and anti-SARS-CoV-2 seroprevalence test results, based on a univariate logistic regression model

\begin{tabular}{|c|c|c|c|c|c|c|c|}
\hline \multirow{2}{*}{$\begin{array}{l}\text { Questions on symptoms occurring between } \\
\text { March 1, } 2020 \text { and } 14 \text { days before blood } \\
\text { donation date }\end{array}$} & \multirow[t]{2}{*}{ Answers } & \multicolumn{2}{|c|}{ Number of individuals (\%) } & \multicolumn{2}{|c|}{$\begin{array}{l}\text { Unadjusted } \\
\text { analysis }\end{array}$} & \multicolumn{2}{|c|}{$\begin{array}{l}\text { Age- and sex-adjusted } \\
\text { analysis }\end{array}$} \\
\hline & & $\begin{array}{l}\text { Antibody-positive } \\
(n=161)\end{array}$ & $\begin{array}{l}\text { Negative controls } \\
(n=162)\end{array}$ & OR & $95 \% \mathrm{CI}$ & OR & $95 \% \mathrm{CI}$ \\
\hline \multirow[t]{2}{*}{$\begin{array}{l}\text { Sick, and presenting symptoms similar } \\
\text { to those reported for COVID-19† }\end{array}$} & $\begin{array}{l}\text { Yes } \\
\text { No }\end{array}$ & $\begin{array}{l}60(37.3) \\
98(60.9)\end{array}$ & $\begin{array}{l}11(6.8) \\
150(92.6)\end{array}$ & \multirow[t]{2}{*}{8.4} & \multirow[t]{2}{*}{$4.2-16.7$} & \multirow[t]{2}{*}{7.9} & \multirow[t]{2}{*}{$3.9-15.8$} \\
\hline & Do not know & $3(1.9)$ & $1(0.6)$ & & & & \\
\hline \multirow[t]{2}{*}{ Fever $\left(\geq 38^{\circ} \mathrm{C}\right)$ or sensation of fever/chills } & $\begin{array}{l}\text { Yes } \\
\text { No }\end{array}$ & $\begin{array}{l}39(24.2) \\
121(75.2)\end{array}$ & $\begin{array}{l}3(1.9) \\
157(96.9)\end{array}$ & \multirow[t]{2}{*}{16.9} & \multirow[t]{2}{*}{$5.1-55.9$} & \multirow[t]{2}{*}{15.1} & \multirow[t]{2}{*}{$4.5-50.3$} \\
\hline & Do not know & $1(0.6)$ & $1(0.6)$ & & & & \\
\hline \multirow[t]{2}{*}{ Weakness } & $\begin{array}{l}\text { Yes } \\
\text { No }\end{array}$ & $\begin{array}{l}36(22.4) \\
125(77.6)\end{array}$ & $\begin{array}{l}6(3.7) \\
155(95.7)\end{array}$ & \multirow[t]{2}{*}{7.4} & \multirow[t]{2}{*}{$3.0-18.2$} & \multirow[t]{2}{*}{7.1} & \multirow[t]{2}{*}{$2.9-17.5$} \\
\hline & Do not know & $0(0.0)$ & $1(0.6)$ & & & & \\
\hline \multirow[t]{2}{*}{ Generalized pain (myalgia, arthralgia) } & $\begin{array}{l}\text { Yes } \\
\text { No }\end{array}$ & $\begin{array}{l}23(14.3) \\
136(84.5)\end{array}$ & $\begin{array}{l}3(1.9) \\
158(97.5)\end{array}$ & \multirow[t]{2}{*}{8.9} & \multirow[t]{2}{*}{$2.6-30.3$} & \multirow[t]{2}{*}{8.4} & \multirow[t]{2}{*}{$2.4-28.8$} \\
\hline & Do not know & $2(1.2)$ & $1(0.6)$ & & & & \\
\hline \multirow[t]{2}{*}{ Rhinorrhea } & $\begin{array}{l}\text { Yes } \\
\text { No }\end{array}$ & $\begin{array}{l}28(17.4) \\
131(81.4)\end{array}$ & $\begin{array}{l}17(10.5) \\
145(89.5)\end{array}$ & \multirow[t]{2}{*}{1.82} & \multirow[t]{2}{*}{$0.95-3.5$} & \multirow[t]{2}{*}{1.76} & \multirow[t]{2}{*}{$0.92-3.4$} \\
\hline & Do not know & $2(1.2)$ & $0(0.0)$ & & & & \\
\hline \multirow[t]{2}{*}{ Sore throat } & $\begin{array}{l}\text { Yes } \\
\text { No }\end{array}$ & $\begin{array}{l}34(21.1) \\
126(78.3)\end{array}$ & $\begin{array}{l}13(8.0) \\
149(92.0)\end{array}$ & \multirow[t]{2}{*}{3.1} & \multirow[t]{2}{*}{$1.56-6.1$} & 2.9 & $1.45-5.8$ \\
\hline & Do not know & $1(0.6)$ & $0(0.0)$ & & & & \\
\hline Cough & $\begin{array}{l}\text { Yes } \\
\text { No }\end{array}$ & $\begin{array}{l}28(17.4) \\
131(81.4)\end{array}$ & $\begin{array}{l}8(4.9) \\
154(95.1)\end{array}$ & 4.1 & $1.81-9.3$ & 4.2 & $1.81-9.5$ \\
\hline & Do not know & $2(1.2)$ & $0(0.0)$ & & & & \\
\hline Shortness of breath/difficulty breathing & $\begin{array}{l}\text { Yes } \\
\text { No }\end{array}$ & $\begin{array}{l}22(13.7) \\
138(85.7)\end{array}$ & $\begin{array}{l}3(1.9) \\
159(98.1)\end{array}$ & 8.5 & $2.5-28.8$ & 8.0 & $2.3-27.5$ \\
\hline & Do not know & $1(0.6)$ & $0(0.0)$ & & & & \\
\hline Loss or reduction in smell or taste sensation & $\begin{array}{l}\text { Yes } \\
\text { No }\end{array}$ & $\begin{array}{l}25(15.5) \\
134(83.2)\end{array}$ & $\begin{array}{l}1(0.6) \\
161(99.4)\end{array}$ & 30.0 & $4.0-224.6$ & 27.6 & $3.7-207.9$ \\
\hline & Do not know & $2(1.2)$ & $0(0.0)$ & & & & \\
\hline Diarrhea & $\begin{array}{l}\text { Yes } \\
\text { No }\end{array}$ & $\begin{array}{l}27(16.8) \\
131(81.4)\end{array}$ & $\begin{array}{l}9(5.6) \\
153(94.4)\end{array}$ & 3.5 & $1.59-7.7$ & 3.5 & $1.57-7.7$ \\
\hline & Do not know & $3(1.9)$ & $0(0.0)$ & & & & \\
\hline At least one symptom $t$ & $\begin{array}{l}\text { Yes } \\
\text { No }\end{array}$ & $\begin{array}{l}84(52.2) \\
77(47.8)\end{array}$ & $\begin{array}{l}31(19.1) \\
131(80.9)\end{array}$ & 4.6 & $2.8-7.6$ & 4.9 & $2.9-8.1$ \\
\hline At least one symptom, excluding rhinorrhea & $\begin{array}{l}\text { Yes } \\
\text { No }\end{array}$ & $\begin{array}{l}80(49.7) \\
81(50.3)\end{array}$ & $\begin{array}{l}29(17.9) \\
133(82.1)\end{array}$ & 4.5 & $2.7-7.5$ & 4.6 & $2.8-7.8$ \\
\hline
\end{tabular}

$O R$, odds ratio

$\dagger$ Individuals for whom symptom onset occurred after blood donation were classified in the "no" group

†Symptoms confirmed following interview with a nurse

The impact of self-exclusion on seroprevalence estimates was explored. Among our 7691 participants, 14 $(0.18 \%)$ seropositive donors reported a diagnosis of confirmed COVID-19, assuming that none of the seronegative donors had been infected. This is lower than the $0.55 \%$ prevalence rate $(31,077$ laboratory-confirmed COVID-19 cases/5.6 million) reported among the 20-69-year-olds by May 25, 2020, when this study started. In the hypothetical scenario where $0.55 \%$ of donors had been diagnosed with
COVID-19 and were antibody-positive, this would have added 28 positive donors $(7691 \times(0.55 \%-0.18 \%))$ to the 173 already detected, and the seroprevalence would increase from $2.23 \%$ to $2.61 \%$.

In univariate analysis, age, history of contact with a COVID-19 case, contact with someone having COVID-19 symptoms, working in the healthcare sector, and living in the Montréal-Laval area were statistically significant risk factors for seropositivity (Table 4). In multivariable parsimonious 
Table 3 Association between healthcare-seeking behaviour and anti-SARS-CoV-2 seroprevalence test results, based on a univariate logistic regression model

\begin{tabular}{|c|c|c|c|c|c|c|c|}
\hline \multirow[t]{2}{*}{$\begin{array}{l}\text { Questions related to healthcare- } \\
\text { seeking behaviour }\end{array}$} & \multirow[t]{2}{*}{ Answers } & \multicolumn{2}{|c|}{$\begin{array}{l}\text { Number of individuals } \\
(\%)\end{array}$} & \multicolumn{2}{|c|}{$\begin{array}{l}\text { Unadjusted } \\
\text { analysis }\end{array}$} & \multicolumn{2}{|c|}{$\begin{array}{l}\text { Age- and sex- } \\
\text { adjusted analy- } \\
\text { sis }\end{array}$} \\
\hline & & $\begin{array}{l}\text { Antibody- } \\
\text { positive }\end{array}$ & $\begin{array}{l}\text { Negative } \\
\text { controls }\end{array}$ & OR & $95 \% \mathrm{CI}$ & OR & $95 \% \mathrm{CI}$ \\
\hline $\begin{array}{l}\text { Have you consulted a physician } \\
\text { regarding your symptoms?† }\end{array}$ & $\begin{array}{l}\text { Yes } \\
\text { No }\end{array}$ & $\begin{array}{l}16(18.6) \ddagger \\
70(81.4)\end{array}$ & $\begin{array}{l}3(9.7) \\
28(90.3)\end{array}$ & 2.1 & $0.58-7.9$ & 1.17 & $0.29-4.8$ \\
\hline \multirow[t]{3}{*}{ Did you have a COVID-19 PCR test? } & Yes, as per the physician's recommendation & $8(29.6)$ & $1(20.0)$ & 2.00 & $0.15-26.7$ & 2.1 & $0.15-14.4$ \\
\hline & $\begin{array}{l}\text { Yes, even though a physician was not consulted } \\
\text { and donor was symptom-free }\end{array}$ & $11(40.7)$ & $2(40.0)$ & 1.38 & $0.16-11.9$ & 1.59 & $0.17-14.4$ \\
\hline & No, as per the physician's recommendation & $8(29.6)$ & $2(40.0)$ & Ref. & Ref. & Ref. & Ref. \\
\hline $\begin{array}{l}\text { What was the COVID-19 PCR test } \\
\text { result? } \dagger\end{array}$ & $\begin{array}{l}\text { Negative } \\
\text { Positive }\end{array}$ & $\begin{array}{l}5(26.3) \\
14(73.7)\end{array}$ & $\begin{array}{l}3(100.0) \\
0(0.0)\end{array}$ & - & - & - & - \\
\hline
\end{tabular}

$O R$, odds ratio; Ref., reference values

$\dagger$ If symptom onset or PCR testing occurred after blood donation, the answer to this question is considered to be "no"

$\$$ Percentages were calculated on the basis of participants who experienced symptoms and includes two donors who were symptom-free

analysis (adjusted for age and sex), the only risk factors significantly associated with antibody positivity were contact with a known COVID-19 case (odds ratio 5.15; 95\% CI
2.04-13.0), contact with a symptomatic individual (odds ratio 2.71; 95\% CI 1.39-5.28), and living in the Montréal-Laval area (odds ratio 2.28; 95\% CI 1.26-4.11). Workplace

Table 4 Association between demographic characteristics and anti-SARS-CoV-2 seroprevalence test results, based on a univariate logistic regression model

\begin{tabular}{|c|c|c|c|c|c|c|c|}
\hline \multirow[t]{2}{*}{ Demographic characteristics } & \multirow[t]{2}{*}{ Values } & \multicolumn{2}{|c|}{ Number of individuals (\%) } & \multicolumn{2}{|c|}{$\begin{array}{l}\text { Unadjusted } \\
\text { analysis }\end{array}$} & \multicolumn{2}{|c|}{$\begin{array}{l}\text { Age- and sex- } \\
\text { adjusted analysis }\end{array}$} \\
\hline & & $\begin{array}{l}\text { Antibody- } \\
\text { positive } \\
(n=161)\end{array}$ & $\begin{array}{l}\text { Negative } \\
\text { controls } \\
(n=162)\end{array}$ & OR & $95 \% \mathrm{CI}$ & OR & $95 \% \mathrm{CI}$ \\
\hline Sex & $\begin{array}{l}\text { Female } \\
\text { Male }\end{array}$ & $\begin{array}{l}88(54.7) \\
73(45.3)\end{array}$ & $\begin{array}{l}72(44.4) \\
90(55.6)\end{array}$ & 1.51 & $0.97-2.34$ & 1.32 & $0.84-2.08$ \\
\hline \multirow[t]{4}{*}{ Age range $\uparrow$ (years) } & $18-24$ & $26(16.2)$ & $17(10.5)$ & 2.49 & $1.15-5.42$ & 2.29 & $1.04-5.04$ \\
\hline & $25-39$ & $46(28.6)$ & $36(22.2)$ & 2.08 & $1.09-3.98$ & 1.89 & $0.97-3.68$ \\
\hline & $40-59$ & $62(38.5)$ & $65(40.1)$ & 1.55 & $0.86-2.81$ & 1.46 & $0.80-2.66$ \\
\hline & $60+$ & $27(16.8)$ & $44(27.2)$ & Ref. & Ref. & Ref. & Ref. \\
\hline $\begin{array}{l}\text { Close contact; with a person diagnosed with } \\
\text { COVID-19 }\end{array}$ & $\begin{array}{l}\text { Yes } \\
\text { No }\end{array}$ & $\begin{array}{l}34(21.1) \\
127(78.9)\end{array}$ & $\begin{array}{l}6(3.7) \\
156(96.3)\end{array}$ & 6.96 & $2.83-17.09$ & 6.12 & $2.46-15.21$ \\
\hline $\begin{array}{l}\text { Close contact with a person not diagnosed with } \\
\text { COVID-19, but who has had cough or fever }\end{array}$ & $\begin{array}{l}\text { Yes } \\
\text { No }\end{array}$ & $\begin{array}{l}42(26.1) \\
119(73.9)\end{array}$ & $\begin{array}{l}16(9.9) \\
146(90.1)\end{array}$ & 3.22 & $1.73-6.02$ & 2.84 & $1.51-5.37$ \\
\hline Healthcare worker§ & $\begin{array}{l}\text { Yes } \\
\text { No }\end{array}$ & $\begin{array}{l}22(13.7) \\
139(86.7)\end{array}$ & $\begin{array}{l}6(3.7) \\
156(96.3)\end{array}$ & 4.12 & $1.62-10.44$ & 3.22 & $1.24-8.37$ \\
\hline \multirow[t]{3}{*}{ Area of residence } & Montréal-Laval & $87(54.0)$ & $59(36.4)$ & 2.59 & $1.51-4.45$ & 2.2 & $1.25-3.86$ \\
\hline & $\begin{array}{l}\text { Surrounding } \\
\text { Montréal-Laval } \\
\text { urban areas }\end{array}$ & $41(25.5)$ & $45(27.8)$ & 1.6 & $0.88-2.92$ & 1.5 & $0.82-2.76$ \\
\hline & Other regions & $33(20.5)$ & $58(35.8)$ & Ref. & Ref. & Ref. & Ref. \\
\hline
\end{tabular}

$O R$, odds ratio; Ref., reference values

$\dagger$ Only 3 donors were more than 69 years old and all were seronegative

\$ Defined as a contact of more than 15 min within $2 \mathrm{~m}$ with no safety protections

$\S$ Defined as any person who works close to ill persons in acute care and chronic care institutions, and first responders; this includes nurses, assistant nurses, physicians, orderlies, and cleaning staff 
Table 5 Exposure context of antibody-positive individuals and negative controls

\begin{tabular}{|c|c|c|c|c|}
\hline \multirow[t]{2}{*}{$\begin{array}{l}\text { In what context might you have been exposed } \\
\text { to a COVID-19-affected person? }\end{array}$} & \multicolumn{2}{|c|}{$\begin{array}{l}\text { Antibody-positive } \\
(n=161)\end{array}$} & \multicolumn{2}{|c|}{$\begin{array}{l}\text { Negative controls } \\
(n=162)\end{array}$} \\
\hline & Numbert & $\%$ & Number $\dagger$ & $\%$ \\
\hline Work & 59 & 36.6 & 32 & 19.8 \\
\hline Home & 32 & 19.9 & 8 & 4.9 \\
\hline Public places & 27 & 16.8 & 17 & 10.5 \\
\hline Travel & $22(1) \ddagger$ & 13.6 & $10(1) \ddagger$ & 6.2 \\
\hline Friends and families & 13 & 8.1 & 20 & 12.3 \\
\hline Leisure activities & 6 & 3.7 & 4 & 2.5 \\
\hline Gatherings & 2 & 1.2 & 0 & 0.0 \\
\hline School/university & 1 & 0.6 & 2 & 1.2 \\
\hline No known exposure/unknown & 33 & 22.4 & 110 & 74.1 \\
\hline
\end{tabular}

$\dagger$ Participants could give more than one answer to the question, which explains why totals exceed the number of participants

$\$$ Numbers in parentheses indicate the number of participants who have been in contact with another traveler
(36.6\%) and home (19.9\%) are the two settings where contacts with a COVID-19 case were most frequently reported by antibody-positive individuals (Table 5).

\section{Discussion}

In the province of Québec, as of May 25, 2020 when our study started, a total of 31,077 laboratory-confirmed COVID-19 cases had been reported among the 20-69-year-olds. This number represents $0.55 \%$ of the 5.6 million people belonging to this age group. Our seroprevalence data in this age group rather suggest that $2.23 \%$ of these adults have been infected by SARS-CoV-2, which is four times more than laboratoryconfirmed COVID-19 cases.

Based on the number of seropositive donors who reported a positive diagnosis of COVID-19 in our nested case-control study, we calculated that this number was three times lower than expected according to the number of cases reported in the general population; we only had 14 such donors, whereas our calculations predicted that we should have had 28 more. The most likely explanation for this discrepancy is that some individuals diagnosed with COVID-19 self-excluded from blood donation, even beyond the mandated 14-day period. This selfexclusion bias would only apply to symptomatic, infected individuals, especially those who had severe enough symptoms to seek medical care. When we calculated the potential impact of this bias on the true seroprevalence rate, the corrected seroprevalence would only be marginally higher, $2.61 \%$ versus $2.23 \%$.

A number of studies reporting anti-SARS-CoV-2 seroprevalence data have been published (Barzin et al., 2020; Biggs et al., 2020; Chen et al., 2020; Hallowell et al., 2020; Kshatri et al., 2020; Lai et al., 2020; Majdoubi et al., 2020; Makaronidis et al., 2020; Murhekar et al., 2020; Picon et al., 2020; Rudberg et al., 2020; Song et al., 2020; Squeri et al., 2020; Stadlbauer et al., 2020; Takita et al., 2020; Wells et al., 2020), including a few with respect to blood donors (Amorim Filho et al., 2020; Erikstrup et al., 2020; Fiore et al., 2020; Fischer et al., 2020; Gallian et al., 2020; $\mathrm{Ng}$ et al., 2020; Percivalle et al., 2020; Younas et al., 2020). Our results are consistent with these reports in that seroprevalence estimates are generally low, albeit often several-fold higher than cumulative COVID-19 incidence rates. In addition to providing an estimate of the burden of the first wave of the pandemic in Québec, our nested case-control study confirms that a substantial proportion of individuals infected with SARS-CoV-2 remain asymptomatic. We also found that fever or sensations of fever/chills, and loss or decline of smell or taste sensation, are most strongly associated with a serological positive test for SARS-CoV-2. Unsurprisingly, contact with a confirmed COVID-19 case or with a symptomatic individual was also predictive of the presence of anti-SARS-CoV-2 antibodies.

Nearly half of antibody-positive individuals did not report any symptom. Among symptomatic, antibody-positive individuals, it is likely that a proportion of those who experienced mild, common cold-like symptoms that are not typical of COVID-19 may have been infected by another virus, as suggested by the fact that $19.1 \%$ of antibody-negative individuals did report similar symptoms. These observations suggest that between half and two thirds of individuals who were infected by SARS-CoV-2 remained asymptomatic. A self-exclusion bias owing to symptomatic, diagnosed COVID-19 cases might have amplified this proportion to some extent. As a side note, thus far there is no evidence that SARS-CoV-2 is blood transmissible (Leblanc et al., 2020). 
This study has some limitations. First, given that the level of SARS-CoV-2-specific antibodies, using RBD or nucleocapsid antigen, tends to wane in some individuals about 100-110 days post-symptom onset (Anand et al., 2020; Perreault et al., 2020; Seow et al., 2020), some cases of previous exposure to SARS-CoV-2 might have been missed; recall that Québec's first documented COVID-19 case was identified in late February 2020. This would lead to an underestimation of the actual seroprevalence rate. However, samples were collected within 4 months of the onset of the first wave of the epidemic in Québec; thus, we would not expect to miss a significant number of cases by loss (waning) of antibodies (seroreversion). It is interesting to note that despite the temporal decline of antibody levels in blood, seropositive individuals may still have immunity, as virus-specific T- and/or memory B-cell responses seem to increase with time and to persist for 6 to 8 months after infection (Dan et al., 2021; Sherina et al., 2020). Second, since the anti-SARS-CoV-2 Spike RBD ELISA is not $100 \%$ sensitive, some antibodypositive individuals might have been missed (Burgess et al., 2020). Conversely, given the very low prevalence of antibodies and a specificity of $98.5 \%$ (with a sensitivity of $98.9 \%$ ) for our in-house assay, some seropositive donors might not have been previously infected by SARS-CoV-2. We decided not to adjust our results for imperfect specificity because we believe our in-house assay likely had better precision than what was estimated, since it was only based on one false-positive event out of 67 negative samples collected before the SARS-CoV-2 outbreak. If seroprevalence is adjusted for specificity, based on the Rogan-Gladen equation (Rogan \& Gladen, 1978), then an overall seroprevalence rate of $1.18 \%(95 \%$ CI 0.86-1.55) is obtained. Finally, participants were blood donors, who may not be perfectly representative of the general population because they are generally healthier. Additionally, a small proportion of participants included plasma donors, who are generally more educated (high school level or higher) and are likely of a higher socioeconomic status (Charbonneau et al., 2015). Furthermore, some of the populations that were more greatly affected by SARS-CoV-2 are less likely to be blood donors, specifically plasma donors. Blood donors may also selfexclude following a COVID-19 infection beyond the mandated 14-day period. As this bias only applies to symptomatically infected individuals who sought medical care, it minimally underestimates the seroprevalence in the general population. However, blood donors can provide convenient samples and are reasonably representative of the healthy adult population. More importantly, donation leftover samples could be used for COVID-19 antibody testing, and large numbers of donations are collected on a daily basis, which allow routine monitoring of seroprevalence over time (O'Brien et al., 2020). Finally, one limitation is the reliance on self-reported health events. Recall bias of such events might have under- or overestimated their true prevalence. Despite these limitations, this study likely provides an accurate estimate of the order of magnitude of the rate of SARS-CoV-2 infection among 18 69-year-old adults in the province of Québec during the first pandemic wave.

\section{Conclusion}

Our results, based on a sample set collected during the first pandemic wave, indicate that despite Québec's being the Canadian province most severely affected by COVID-19, SARS-CoV-2 seroprevalence in Québec, though much higher than the cumulative COVID-19 incidence, was still relatively low, and far from the level of herd immunity needed to limit viral spread.

\section{Contributions to knowledge}

What does this study add to existing knowledge?

- Although the number of confirmed COVID-19 cases identified by healthcare systems is one indicator of the progression of the pandemic, the true burden of infection can be more precisely estimated by SARS-CoV-2 antibody seroprevalence in the general population.

- Few studies in a general population setting have correlated the presence or absence of anti-SARS-CoV-2 antibodies with the occurrence of COVID-19 symptoms, and none of these studies have used a control group to estimate the frequency of asymptomatic SARS-CoV-2 infections in this population.

- Our study advances understanding of the epidemiology of COVID-19.

What are the key implications for public health interventions, practice, or policy?

- Based on blood donor samples collected after the first pandemic wave, this study shows that, in Québec, which had the highest rate of PCR-confirmed COVID19 infections in Canada, less than 3\% of 18-69-year-old adults have been infected, which is relatively low, and far from the level of herd immunity needed to limit viral spread.

- The fact that three out of four infections escaped detection by public health illustrates the limitations of current control policies based on contact tracing of confirmed cases.

Supplementary Information The online version contains supplementary material available at https://doi.org/10.17269/s41997-021-00531-6. 
Acknowledgements The authors are grateful to the blood donors who participated in this study and the Héma-Québec team involved in this project. We also thank Jean-François Leblanc at Héma-Québec for his help in writing the manuscript.

Availability of data and material Data and material are available upon request.

\section{Code availability Code is available upon request.}

Author contributions AL, GD, GDS, RB, MG: conceptualization; formal analysis, methodology; writing - original draft. RT: project coordination, manuscript revision. AF, AG, GBD, FB: methodology; writing - review and editing. JP, MD, MJF, TT, JB, GBD, JP, GGL: laboratory analysis; writing - review and editing.

Funding This work was supported by le Ministère de la Santé et des Services Sociaux du Québec.

\section{Declarations}

Ethics approval This study was approved by Héma-Québec's Research Ethics Committee.

Consent to participate Participants consented to participate in this study.

Consent for publication Publication was foreseen in the acceptance of participation.

Conflict of interest The authors declare no competing interests.

Disclaimer The funders had no role in study design, data collection and analysis, decision to publish, or preparation of the manuscript.

\section{References}

Amorim Filho, L., Szwarcwald, C. L., Mateos, S., O. G., Leon, A. C. M. P., de Medronho, R., A., Veloso, V. G., Lopes, J. I. F., de Porto, L. C., M. S., Chieppe, A., Werneck, G. L., \& Grupo Hemorio de Pesquisa em Covid-19. (2020). Seroprevalence of anti-SARSCoV-2 among blood donors in Rio de Janeiro, Brazil. Revista De Saude Publica, 54, 69. https://doi.org/10.11606/s1518-8787. 2020054002643

Anand, S. P., Prévost, J., Richard, J., Perreault, J., Tremblay, T., Drouin, M., Fournier, M.-J., Lewin, A., Bazin, R., \& Finzi, A. (2020). Highthroughput detection of antibodies targeting the SARS-CoV-2 Spike in longitudinal convalescent plasma samples. BioRxiv, 2020(10), 20.346783. https://doi.org/10.1101/2020.10.20.346783.

Barzin, A., Schmitz, J. L., Rosin, S., Sirpal, R., Almond, M., Robinette, C., Wells, S., Hudgens, M., Olshan, A., Deen, S., Krejci, P., Quackenbush, E., Chronowski, K., Cornaby, C., Goins, J., Butler, L., Aucoin, J., Boyer, K., Faulk, J., et al. (2020). SARS-CoV-2 seroprevalence among a Southern U.S. population indicates limited asymptomatic spread under physical distancing measures. MBio, 11(5), e02426-e02420. https://doi.org/10.1128/mBio.02426-20.

Biggs, H. M., Harris, J. B., Breakwell, L., Dahlgren, F. S., Abedi, G. R., Szablewski, C. M., Drobeniuc, J., Bustamante, N. D., Almendares, O., Schnall, A. H., Gilani, Z., Smith, T., Gieraltowski, L., Johnson, J. A., Bajema, K. L., McDavid, K., Schafer, I. J., Sullivan, V., Punkova, L., et al. (2020). Estimated community seroprevalence of SARS-CoV-2 antibodies-Two Georgia counties, April 28May 3, 2020. MMWR. Morbidity and Mortality Weekly Report, 69(29), 965-970. https://doi.org/10.15585/mmwr.mm6929e2.

Burgess, S., Ponsford, M. J., \& Gill, D. (2020). Are we underestimating seroprevalence of SARS-CoV-2? BMJ (Clinical Research Ed.), 370, m3364. https://doi.org/10.1136/bmj.m3364.

Center for Systems Science and Engineering (CSSE). (2020). COVID-19 dashboard. Hopkins University (JHU). https://gisanddata.maps. a r c gis .com/apps/opsdashboard/index.html\#/ bda7594740fd40299423467b48e9ecf6.

Charbonneau, J., Cloutier, M.-S., \& Carrier, É. (2015). Whole blood and apheresis donors in Quebec, Canada: Demographic differences and motivations to donate. Transfusion and Apheresis Science : Official Journal of the World Apheresis Association : Official Journal of the European Society for Haemapheresis, 53(3). https://doi.org/10. 1016/j.transci.2015.06.001.

Chen, X., Chen, Z., Azman, A. S., Deng, X., Chen, X., Lu, W., Zhao, Z., Yang, J., Viboud, C., Ajelli, M., Leung, D. T., \& Yu, H. (2020). Serological evidence of human infection with SARS-CoV-2: A systematic review and meta-analysis. MedRxiv: The Preprint Server for Health Sciences. https://doi.org/10.1101/2020.09.11.20192773.

Dan, J. M., Mateus, J., Kato, Y., Hastie, K. M., Yu, E. D., Faliti, C. E., Grifoni, A., Ramirez, S. I., Haupt, S., Frazier, A., Nakao, C., Rayaprolu, V., Rawlings, S. A., Peters, B., Krammer, F., Simon, V., Saphire, E. O., Smith, D. M., Weiskopf, D., et al. (2021). Immunological memory to SARS-CoV-2 assessed for up to 8 months after infection. Science. https://doi.org/10.1126/science. abf4063.

Erikstrup, C., Hother, C. E., Pedersen, O. B. V., Mølbak, K., Skov, R. L., Holm, D. K., Sækmose, S. G., Nilsson, A. C., Brooks, P. T., Boldsen, J. K., Mikkelsen, C., Gybel-Brask, M., Sørensen, E., Dinh, K. M., Mikkelsen, S., Møller, B. K., Haunstrup, T., Harritshøj, L., Jensen, B. A., et al. (2020). Estimation of SARSCoV-2 infection fatality rate by real-time antibody screening of blood donors. Clinical Infectious Diseases : An Official Publication of the Infectious Diseases Society of America. https:// doi.org/10.1093/cid/ciaa849.

European Centre for Disease Prevention and Control (ECDC). (2020). COVID-19 situation update worldwide. European Centre for Disease Prevention and Control (ECDC). https://www.ecdc. europa.eu/en/geographical-distribution-2019-ncov-cases.

Fiore, J. R., Centra, M., De Carlo, A., Granato, T., Rosa, A., Sarno, M., De Feo, L., Di Stefano, M., Errico, M. D., Caputo, S. L., De Nittis, R., Arena, F., Corso, G., Margaglione, M., \& Santantonio, T. A. (2020). Results from a survey in healthy blood donors in South Eastern Italy indicate that we are far away from herd immunity to SARS-CoV-2. Journal of Medical Virology, 93(3), 17391742. https://doi.org/10.1002/jmv.26425.

Fischer, B., Knabbe, C., \& Vollmer, T. (2020). SARS-CoV-2 IgG seroprevalence in blood donors located in three different federal states, Germany, March to June 2020. Euro Surveillance: Bulletin Europeen Sur Les Maladies Transmissibles = European Communicable Disease Bulletin, 25(28). https://doi.org/10.2807/ 1560-7917.ES.2020.25.28.2001285.

Gallian, P., Pastorino, B., Morel, P., Chiaroni, J., Ninove, L., \& de Lamballerie, X. (2020). Lower prevalence of antibodies neutralizing SARS-CoV-2 in group O French blood donors. Antiviral Research, 181, 104880. https://doi.org/10.1016/j.antiviral.2020.104880.

Hallowell, B. D., Carlson, C. M., Jacobs, J. R., Pomeroy, M., Steinberg, J., Tenforde, M. W., McDonald, E., Foster, L., Feldstein, L. R., Rolfes, M. A., Haynes, A., Abedi, G. R., Odongo, G. S., Saruwatari, K., Rider, E. C., Douville, G., Bhakta, N., Maniatis, P., Lindstrom, S., et al. (2020). Severe acute respiratory syndrome coronavirus 2 prevalence, seroprevalence, and exposure among evacuees from Wuhan, China, 2020. Emerging Infectious 
Diseases, 26(9), 1998-2004. https://doi.org/10.3201/eid2609. 201590.

Heffron, A. S., McIlwain, S. J., Baker, D. A., Amjadi, M. F., Khullar, S., Sethi, A. K., Shelef, M. A., O’Connor, D. H., \& Ong, I. M. (2020). The landscape of antibody binding to SARS-CoV-2. BioRxiv. https://doi.org/10.1101/2020.10.10.334292.

Institut national de santé publique du Québec. (n.d.-a). Données COVID19 au Québec. INSPQ. https://www.inspq.qc.ca/covid-19/donnees. Accessed 2 November 2020.

Institut national de santé publique du Québec. (n.d.-b). Données COVID19-Ensemble des régions du Québec. INSPQ. https://www.inspq. qc.ca/covid-19/donnees/regions. Accessed 16 October 2020.

Kshatri, J. S., Bhattacharya, D., Kanungo, S., Giri, S., Palo, S. K., Parai, D., Turuk, J., Mansingh, A., Choudhary, H., Dash, G. C., Mishra, N., Satapathy, D. M., Sahoo, S. K., \& Pati, S. (2020). Findings from serological surveys (in August 2020) to assess the exposure of adult population to SARS Cov-2 infection in three cities of Odisha, India. MedRxiv, 2020(10), 11.20210807. https://doi.org/10.1101/2020.10. 11.20210807.

Lai, C.-C., Wang, J.-H., \& Hsueh, P.-R. (2020). Population-based seroprevalence surveys of anti-SARS-CoV-2 antibody: An up-to-date review. International Journal of Infectious Diseases: IJID: Official Publication of the International Society for Infectious Diseases. https://doi.org/10.1016/j.ijid.2020.10.011.

Leblanc, J.-F., Germain, M., Delage, G., \& O Brien, S., Drews, S. J., \& Lewin, A. (2020). Risk of transmission of severe acute respiratory syndrome coronavirus 2 by transfusion: A literature review. Transfusion, 60(12), 3046-3054. https://doi.org/10.1111/trf.16056.

Majdoubi, A., O'Connell, S. E., Michalski, C., Dada, S., Narpala, S., Gelinas, J., Mehta, D., Cheung, C., Basappa, M., Gorges, M., Barakauskas, V. E., Goldfarb, D. M., Douek, D. C., McDermott, A. B., \& Lavoie, P. M. (2020). Antibody reactivity to SARS-CoV-2 in adults from the Vancouver metropolitan area Canada. MedRxiv: The Preprint Server for Health Sciences. https://doi.org/10.1101/ 2020.10.05.20206664.

Makaronidis, J., Mok, J., Balogun, N., Magee, C. G., Omar, R. Z., Carnemolla, A., \& Batterham, R. L. (2020). Seroprevalence of SARS-CoV-2 antibodies in people with an acute loss in their sense of smell and/or taste in a community-based population in London, UK: An observational cohort study. PLoS Medicine, 17(10), e1003358. https://doi.org/10.1371/journal.pmed.1003358.

Mizumoto, K., Kagaya, K., Zarebski, A., \& Chowell, G. (2020). Estimating the asymptomatic proportion of coronavirus disease 2019 (COVID-19) cases on board the Diamond Princess cruise ship, Yokohama, Japan, 2020. Euro Surveillance: Bulletin Europeen Sur Les Maladies Transmissibles $=$. European Communicable Disease Bulletin, 25(10). https://doi.org/10.2807/1560-7917.ES.2020.25.10. 2000180.

Murhekar, M. V., Bhatnagar, T., Selvaraju, S., Rade, K., Saravanakumar, V., Vivian Thangaraj, J. W., Kumar, M. S., Shah, N., Sabarinathan, R., Turuk, A., Anand, P. K., Asthana, S., Balachandar, R., Bangar, S. D., Bansal, A. K., Bhat, J., Chakraborty, D., Rangaraju, C., Chopra, V., et al. (2020). Prevalence of SARS-CoV-2 infection in India: Findings from the national serosurvey, May-June 2020. The Indian Journal of Medical Research, 152(1 \& 2), 48-60. https://doi. org/10.4103/ijmr.IJMR_3290_20.

Ng, D. L., Goldgof, G. M., Shy, B. R., Levine, A. G., Balcerek, J., Bapat, S. P., Prostko, J., Rodgers, M., Coller, K., Pearce, S., Franz, S., Du, L., Stone, M., Pillai, S. K., Sotomayor-Gonzalez, A., Servellita, V., Martin, C. S. S., Granados, A., Glasner, D. R., et al. (2020). SARS$\mathrm{CoV}-2$ seroprevalence and neutralizing activity in donor and patient blood. Nature Communications, 11(1), 4698. https://doi.org/10. 1038/s41467-020-18468-8.

Nogrady, B. (2020). What the data say about asymptomatic COVID infections. Nature, 587(7835), 534-535. https://doi.org/10.1038/ d41586-020-03141-3.
O’Brien, S. F., Lieshout-Krikke, R. W., Lewin, A., Erikstrup, C., Steele, W. R., Uzicanin, S., Custer, B., \& Surveillance, Risk Assessment, Policy Sub-group of the ISBT Transfusion Transmitted Infectious Diseases Working Party. (2020). Research initiatives of blood services worldwide in response to the covid-19 pandemic. Vox Sanguinis, 116, 296-304. https://doi.org/10.1111/vox.12995.

Percivalle, E., Cambiè, G., Cassaniti, I., Nepita, E. V., Maserati, R., Ferrari, A., Di Martino, R., Isernia, P., Mojoli, F., Bruno, R., Tirani, M., Cereda, D., Nicora, C., Lombardo, M., \& Baldanti, F. (2020). Prevalence of SARS-CoV-2 specific neutralising antibodies in blood donors from the Lodi Red Zone in Lombardy, Italy, as at 06 April 2020. Euro Surveillance: Bulletin Europeen Sur Les Maladies Transmissibles $=$ European Communicable Disease Bulletin, 25(24). https://doi.org/10.2807/1560-7917.ES.2020.25.24. 2001031.

Perreault, J., Tremblay, T., Fournier, M.-J., Drouin, M., BeaudoinBussières, G., Prévost, J., Lewin, A., Bégin, P., Finzi, A., \& Bazin, R. (2020). Waning of SARS-CoV-2 RBD antibodies in longitudinal convalescent plasma samples within four months after symptom onset. Blood, 136(22), 2588-2591. https://doi.org/10. 1182/blood.2020008367.

Picon, R. V., Carreno, I., da Silva, A. A., Mossmann, M., Laste, G., \& Domingues, G. de C., Heringer, L. F. F., Gheno, B. R., Alvarenga, L. L., \& Conte, M. (2020). Coronavirus disease 2019 populationbased prevalence, risk factors, hospitalization, and fatality rates in southern Brazil. International Journal of Infectious Diseases: IJID: Official Publication of the International Society for Infectious Diseases, 100, 402-410. https://doi.org/10.1016/j.ijid.2020.09.028.

Prévost, J., Gasser, R., Beaudoin-Bussières, G., Richard, J., Duerr, R., Laumaea, A., Anand, S. P., Goyette, G., Benlarbi, M., Ding, S., Medjahed, H., Lewin, A., Perreault, J., Tremblay, T., GendronLepage, G., Gauthier, N., Carrier, M., Marcoux, D., Piché, A., et al. (2020). Cross-sectional evaluation of humoral responses against SARS-CoV-2 spike. Cell Reports. Medicine, 100126. https://doi.org/10.1016/j.xcrm.2020.100126.

Qu, J., Wu, C., Li, X., Zhang, G., Jiang, Z., Li, X., Zhu, Q., \& Liu, L. (2020). Profile of IgG and IgM antibodies against severe acute respiratory syndrome coronavirus 2 (SARS-CoV-2). Clinical Infectious Diseases: An Official Publication of the Infectious Diseases Society of America, ciaa489 71(16), 2255-2258. https:// doi.org/10.1093/cid/ciaa489.

Rogan, W. J., \& Gladen, B. (1978). Estimating prevalence from the results of a screening test. American Journal of Epidemiology, 107(1), 71-76. https://doi.org/10.1093/oxfordjournals.aje.a112510.

Rudberg, A.-S., Havervall, S., Månberg, A., Jernbom Falk, A., Aguilera, K., Ng, H., Gabrielsson, L., Salomonsson, A.-C., Hanke, L., Murrell, B., McInerney, G., Olofsson, J., Andersson, E., Hellström, C., Bayati, S., Bergström, S., Pin, E., Sjöberg, R., Tegel, H., et al. (2020). SARS-CoV-2 exposure, symptoms and seroprevalence in healthcare workers in Sweden. Nature Communications, 11(1), 5064. https://doi.org/10.1038/s41467020-18848-0.

Seow, J., Graham, C., Merrick, B., Acors, S., Pickering, S., Steel, K. J. A., Hemmings, O., O’Byrne, A., Kouphou, N., Galao, R. P., Betancor, G., Wilson, H. D., Signell, A. W., Winstone, H., Kerridge, C., Huettner, I., Jimenez-Guardeño, J. M., Lista, M. J., Temperton, N., et al. (2020). Longitudinal observation and decline of neutralizing antibody responses in the three months following SARS-CoV-2 infection in humans. Nature Microbiology, 5(12), 1598-1607. https://doi.org/10.1038/s41564-020-00813-8.

Sherina, N., Piralla, A., Du, L., Wan, H., Kumagai-Braesh, M., Andréll, J., Braesch-Andersen, S., Cassaniti, I., Percivalle, E., Sarasini, A., Bergami, F., Martino, R. D., Colaneri, M., Vecchia, M., Sambo, M., Zuccaro, V., Bruno, R., Oggionni, T., Meloni, F., et al. (2020). Persistence of SARS-CoV-2 specific B- and T-cell responses in convalescent COVID-19 patients 6-8 months after the infection. 
BioRxiv, 2020(11), 06.371617. https://doi.org/10.1101/2020.11.06. 371617.

Shrock, E., Fujimura, E., Kula, T., Timms, R. T., Lee, I.-H., Leng, Y., Robinson, M. L., Sie, B. M., Li, M. Z., Chen, Y., Logue, J., Zuiani, A., McCulloch, D., Lelis, F. J. N., Henson, S., Monaco, D. R., Travers, M., Habibi, S., Clarke, W. A., et al. (2020). Viral epitope profiling of COVID-19 patients reveals cross-reactivity and correlates of severity. Science. New York. https://doi.org/10.1126/science. abd4250.

Song, S. K., Lee, D. H., Nam, J. H., Kim, K. T., Do, J. S., Kang, D. W., Kim, S. G., \& Cho, M. R. (2020). IgG seroprevalence of COVID-19 among individuals without a history of the coronavirus disease infection in Daegu, Korea. Journal of Korean Medical Science, 35(29), e269. https://doi.org/10.3346/jkms.2020.35.e269.

Squeri, R., Levita, A., Intelisano, R., Costa, G. B., Mancuso, G., Grasso, L., D’Amato, S., Mazzitelli, F., Squeri, A., Midiri, A., Biondo, C., Alesci, D., Bonaccorso, V., Bitto, A., \& Genovese, C. (2020). Correct management and low rate of contagiousness of healthcare workers in a University Hospital in Southern Italy: From contact tracing to serological investigation. Acta Bio-Medica: Atenei Parmensis, 91(9-S), 79-86. https://doi.org/10.23750/abm.v91i9-S. 10118.

Stadlbauer, D., Tan, J., Jiang, K., Hernandez, M. M., Fabre, S., Amanat, F., Teo, C., Arunkumar, G. A., McMahon, M., Capuano, C., Twyman, K., Jhang, J., Nowak, M. D., Simon, V., Sordillo, E. M., van Bakel, H., \& Krammer, F. (2020). Repeated cross-sectional sero-monitoring of SARS-CoV-2 in New York City. Nature. https://doi.org/10.1038/s41586-020-2912-6.

Takita, M., Matsumura, T., Yamamoto, K., Yamashita, E., Hosoda, K., Hamaki, T., \& Kusumi, E. (2020). Geographical profiles of COVID-19 outbreak in Tokyo: An analysis of the primary care clinic-based point-of-care antibody testing. Journal of Primary Care \& Community Health, 11, 2150132720942695. https://doi. org/10.1177/2150132720942695.
Wells, P. M., Doores, K. J., Couvreur, S., Nunez, R. M., Seow, J., Graham, C., Acors, S., Kouphou, N., Neil, S. J. D., Tedder, R. S., Matos, P. M., Poulton, K., Lista, M. J., Dickenson, R. E., Sertkaya, H., Maguire, T. J. A., Scourfield, E. J., Bowyer, R. C. E., Hart, D., et al. (2020). Estimates of the rate of infection and asymptomatic COVID-19 disease in a population sample from SE England. The Journal of Infection. https://doi.org/10.1016/j.jinf.2020.10.011.

Worldometer. (2020). COVID-19 virus pandemic. Worldometer. https:// www.worldometers.info/coronavirus/.

Wu, Z., \& McGoogan, J. M. (2020). Characteristics of and important lessons from the coronavirus disease 2019 (COVID-19) outbreak in China: Summary of a report of 72314 cases from the Chinese Center for Disease Control and Prevention. JAMA 2020, 323(13), 1239-1242. https://doi.org/10.1001/jama.2020.2648.

Younas, A., Waheed, S., Khawaja, S., Imam, M., Borhany, M., \& Shamsi, T. (2020). Seroprevalence of SARS-CoV-2 antibodies among healthy blood donors in Karachi. Pakistan. Transfusion and Apheresis Science: Official Journal of the World Apheresis Association: Official Journal of the European Society for Haemapheresis, 102923. https://doi.org/10.1016/j.transci.2020. 102923.

Zhao, J., Yuan, Q., Wang, H., Liu, W., Liao, X., Su, Y., Wang, X., Yuan, J., Li, T., Li, J., Qian, S., Hong, C., Wang, F., Liu, Y., Wang, Z., He, Q., Li, Z., He, B., Zhang, T., et al. (2020). Antibody responses to SARS-CoV-2 in patients of novel coronavirus disease 2019. Clinical Infectious Diseases : An Official Publication of the Infectious Diseases Society of America, ciaa344. 590(7844), 146150. https://doi.org/10.1093/cid/ciaa344.

Publisher's note Springer Nature remains neutral with regard to jurisdictional claims in published maps and institutional affiliations. 